\title{
Effects and Mechanisms of Checkpoint Inhibitors (CTLA-4, PD-1 and PD-L1 Inhibitors) as New Immunotherapeutic Agents for Bladder Cancer
}

\author{
(D) Serdar Çelik MD ${ }^{1,2}$, (D) Zekiye Sultan Altun MD², (D) Safiye Aktaş MD² \\ I'zmir Bozyaka Training and Research Hospital, Clinic of Urology, Izmir, Turkey \\ ${ }^{2}$ Dokuz Eylül University, Institute of Oncology, Department of Basic Oncology, Izmir, Turkey
}

\begin{abstract}
Since intravesical Bacillus Calmette-Guerin (BCG) began to be used for bladder cancer, our understanding of the importance of immune mechanisms in bladder cancer has steadily grown. With developments in immunotherapy in recent years, the use of new immunotherapeutic agents for bladder cancer, especially chemotherapy-resistant invasive and metastatic cancers, has opened the way for research in this area. Of these new therapeutic agents, this article reviews studies published on PubMed or listed on the ClinicalTrials.gov website as of December 2017 regarding the effects and mechanisms of action of checkpoint inhibitors [cytotoxic t-lymphocyte associated protein-4, programmed cell death 1 receptor (PD-1) and PD-1 ligand inhibitors] on bladder cancer. Because checkpoint inhibitors were first used for chemotherapy-resistant bladder cancer after identification of positive expression in tumor cells and especially in tumor-infiltrating mononuclear cells, significant objective response rates and survival advantages have been reported. Research continues regarding the use of these agents as first- and second-line treatment for metastatic disease in combination with chemotherapy; their efficacy in neoadjuvant, adjuvant, and bladder-preserving approaches to muscle-invasive bladder cancer (MIBC) disease, and their use in non-muscle-invasize bladder cancer (NMIBC), especially BCG-refractory disease. Depending on the results of these ongoing studies, immunotherapy may direct the treatment of bladder cancer in the future.
\end{abstract}

Keywords: Bladder cancer, immunotherapy, PD-1, PD-L1, CTLA-4

\section{Introduction}

Bladder cancer ranks fifth among the most common types of cancer (1). Seventy-five percent of cases are identified as nonmuscle-invasive bladder cancers (NMIBC) [(pathologic stage Ta, $\mathrm{T} 1$ and carcinoma in situ (CIS)] following transurethral resection. European Organization for Research and Treatment of Cancer risk groups have been defined to predict the recurrence and progression of NMIBC, and follow-up and treatment protocols are recommended based on this classification (2). Intravesical Bacillus Calmette-Guerin (BCG) therapy is the only agent that reduces recurrence and progression to muscle-invasive bladder cancer (MIBC), and is especially recommended for high-risk patients $(2,3)$. The search for alternative treatments is ongoing due to the risk of toxicity or unresponsiveness to BCG therapy. Immunotherapy is the most important and newest of these research areas, yet is also the oldest (because it forms the basis of the BCG mechanism of action).
There are several reasons that immunotherapy is a favorable treatment modality for bladder cancer. Firstly, bladder cancer has one of the highest mutation rates among all cancers. Therefore, it has high antigenic potential (4). Secondly, because the tumor is surrounded by a large surface in the intravesical area, it is easily accessible and suitable for local treatment. Thirdly, follow-up is easy because response to treatment can be observed visually. Despite these advantages, however, treatment success in bladder cancer is not at the desired level. For this reason, it is also an important target in research on new immunotherapeutic agents (5).

The aim of this review is to discuss bladder cancer immunology and the role of new immunotherapeutic agents (inhibitors) in the treatment of bladder cancer in light of the current literature. The contents are presented within the following subheadings: Immunotherapy in bladder cancer, BCG and bladder cancer, and checkpoint inhibition and inhibitors. Substantial attention 
is given to the most studied group of compounds, checkpoint inhibitors, in the treatment of locally invasive and metastatic bladder cancers. This is followed by sections concerning the role of checkpoint inhibitors in the neoadjuvant and bladderpreserving approach to MIBC and their role in the treatment of NMIBC. Finally, we present checkpoint expressions in urothelial tumors.

\section{The Basis of Immunotherapy in Bladder Cancer}

While the relationship between the immune system and the foundations of neoplasia has been known since 1891, BCG was shown to be an effective agent in the treatment of bladder cancer by Morales et al. (6) in 1976. BCG has been used in the treatment of bladder cancer since that time and is still, over 40 years later, recommended for the treatment of high-risk NMIBC.

\section{Bacillus Calmette-Guerin and Bladder Cancer}

Calmette et al. (7) developed BCG from Mycobacterium bovis as an antituberculosis vaccine. However, after the link between malignancy and the immune system was established, BCG began to be used in the treatment of cancer. After showing efficacy against implanted tumors in mice, BCG was used in leukemia, melanoma, and head and neck cancers; it was first applied in the bladder as endoscopic intralesional injection for melanoma metastasis $(8,9)$. Later, Morales et al. (6) demonstrated the efficacy of intravesical BCG in patients with NMIBC. In their study, which was the first to describe the use of BCG in bladder cancer, patients who had frequent recurrence and could not undergo total resection were treated with 120 $\mathrm{mg}$ intravesical BCG and intradermal BCG injections for 6 weeks (6). Since the first studies $(6,10)$, BCG has becommen a standard therapeutic agent in use from the 1990s to the present, especially for patients with high-risk NMIBC.

The mechanism of action of BCG is based on immune system activation and the immune response. The immune response begins with the macropinocytosis of BCG into the urothelial cells, followed by the upregulation of major histocompatibility complex class 2 molecules and cytokine release. This results in migration of Th1 lymphocytes to the area around the tumor and the formation of a cytotoxic immune response mediated by CD8+ lymphocytes, natural killer (NK) cells, and granulocytes (11).

\section{Checkpoint Inhibition and Inhibitors}

Immune checkpoint inhibition is at the forefront of current cancer research. It was approved by the United States Food and Drug Administration (FDA) following positive results from phase 3 trials on checkpoint inhibition in melanoma, non-small cell lung cancer, and renal cell carcinoma. However, the focus of research is inverse to severity in urinary system malignancies, especially urothelial carcinoma. Most of the research and FDA approvals related to checkpoint inhibition pertain to the locally invasive and metastatic patient groups. Therefore, we divided studies investigating checkpoint inhibitors in urothelial carcinoma into those focusing on locally invasive and metastatic bladder cancer in Table 1, MIBC in Table 2, and NMIBC in Table 3.
The mechanism of checkpoint inhibition targets T cell regulation, increasing $T$ cell and antitumor activity by suppressing inhibitor signals. This shows that, in addition to the previously known $T$ cell receptor (TCR) activation, there are many co-stimulatory and inhibitory molecules on the surface of $\mathrm{T}$ cells and that these influence $T$ cell behavior $(12,13)$. The most important of these are cytotoxic T-lymphocyte associated protein-4 (CTLA-4) and programmed cell death 1 receptor (PD-1) and its ligands (PDL1 and PD-L2) (Figure 1), which will be discussed in detail in the next section.

\section{Cytotoxic T-Lymphocyte Associated Protein-4 and Ipilimumab (CTLA-4 Inhibitor)}

CTLA-4 expressed on the surface of T cells is known to be among the molecules involved in T cell activation. CTLA-4 competes with CD28, an immunostimulant receptor, for B7 ligands (B7-1 and B7-2) found on the surface of antigenpresenting cells (APCs). However, the B7/CTLA-4 complex inhibits $T$ cell activation in the lymphoid tissue instead of enhancing it as the CD28 complex does. This shows that CTLA4 inhibition can promote an immune response. Therefore, when the B7/CTLA-4 interaction is blocked by ipilimumab, a monoclonal anti-CTLA-4 antibody, the T cell balance is shifted towards activation, increasing the antitumor effects. Ipilimumab first received FDA approval for metastatic melanoma (14). In one large trial, 799 patients with metastatic castration-resistant prostate cancer that progressed after docetaxel chemotherapy for urologic malignancies were given a placebo or ipilimumab following external radiotherapy applied to the bone (15). While no difference in overall survival was observed between the groups, ipilimumab yielded more favorable outcomes in terms of prostate-specific antigen reduction and progression-free

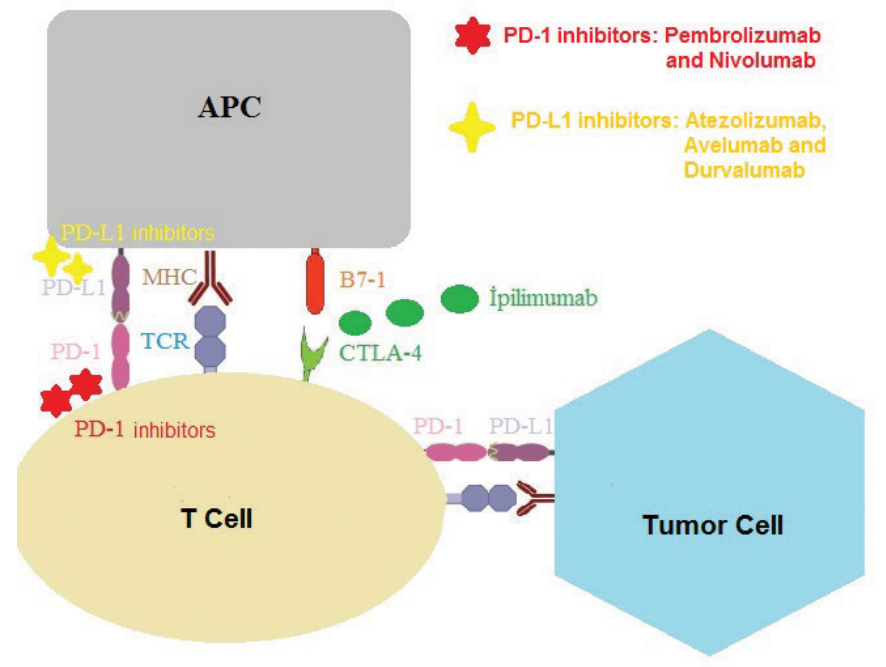

Figure 1. The receptor-ligand relationship in checkpoint inhibition, and the cells and checkpoints affected by inhibitors: cytotoxic T-lymphocyte associated protein-4, programmed cell death 1 receptor, and programmed cell death 1 ligand CTLA-4: Cytotoxic T-lymphocyte associated protein-4, PD-L1: Programmed cell death 1 ligand, PD-1: Programmed cell death 1 receptor, APC: Antigen-presenting cell, TCR: T cell receptor 
survival ( 4 months vs 3.1 months). In another study, 12 patients whose clinical stage was T1-T2N0M0 were given 2 doses of ipilimumab prior to cystectomy and side effects resulted in delayed cystectomy in 2 patients (16). Phase 2 trials evaluating the combination of ipilimumab with gemcitabine and cisplatin in patients with advanced disease are ongoing. Side effects of ipilimumab include vitiligo, rashes, pruritus, anorexia, fatigue, diarrhea, and in a small number of patients, immune-related effects requiring steroid treatment (14). Trials of ipilimumab conducted in the locally invasive and metastatic patient group are presented in Table 1.

\section{Programmed Cell Death 1 Receptor and Its Ligands (PD-L1 and PD-L2)}

PD-1 receptor (CD279) and its two ligands PD-1 ligand 1 (PDL1, B7-H1, and CD274) and PD-1 ligand 2 (PD-L2, B7-DC, and CD273) are cell surface glycoproteins from the B7 family of coinhibitory molecules. PD-L1 is found on the surfaces of APCs, T cells, NK cells, stem cells, and various non-hematopoietic cells in humans (17). PD-L2 has been shown in a few studies to be expressed in a small number of cells. PD-L1 and PD-L2 bind to the PD-1 receptor expressed by T cells, and these ligands are also found in APCs such as macrophages, dendritic cells, and $B$ cells. This receptor and its ligands are important molecules involved in $\mathrm{T}$ cell immunomodulation. The PD-1 receptor inhibits TCR-mediated T cell function, as does CTLA-4. However, unlike CTLA-4, they are believed to exert this effect in the tumor microenvironment (18). Upregulation of PD-L1 in tumor cells is considered a mechanism of PD-1 pathway activation and immune escape (19). Indeed, immunohistochemical studies have shown that increased PD-L1 expression is associated with advanced stage bladder cancer and high-grade tumors (20). Therefore, the following sections include a detailed discussion of studies investigating the effect of inhibitory drugs that target PD-1 and PD-L1 in bladder cancer.

\section{Atezolizumab (PD-L1 Inhibitor)}

Atezolizumab, a monoclonal immunoglobulin G1 antibody that binds PD-L1, came into use following FDA approval in the treatment of advanced stage bladder cancer that progresses despite platinum-based chemotherapy $(12,21)$. This approval was obtained by examining data obtained from phase 1 and 2 trials and based on the presence of PD-L1 in tissue samples taken from advanced stage patients prior to treatment. In a phase 1 trial, response was observed in $25 \%$ of the patients, with 2 patients showing complete response. However, this response was found to rely not on the immunohistochemical scores of tumor cells, but rather on the scores of tumor infiltrating mononuclear cells (TIMCs). In a phase 1 trial involving 68 patients with metastatic urothelial carcinoma who had received prior treatment (93\% had previously undergone cisplatin-based chemotherapy; systemic therapy failed in 72\%), the objective response rate at 6-week follow-up was 50\% among those with a high PD-L1 expression in the TIMCs, compared to only 8.3\% among those who were PD-L1 negative. Overall, 57\% of patients experienced a side effect such as anorexia, fatigue, nausea, weakness, and shivering $(12,13)$. This was followed by the results of the phase 2 IMvigor210 (NCT02108652) study (21). In that trial, 310 inoperable and metastatic patients with an Eastern Cooperative Oncology Group performance score of 0 or 1 were evaluated. PD-L1 expression in TIMCs was determined using SP142 assay. Tumors were grouped according to expression rate: $<1 \%$; $\geq 1 \%$ to $<5 \%$; and $\geq 5 \%$. The overall response rate was $15 \%$ among the 310 patients, and response was found to be associated with expression rates (higher response rate with higher TIMC PD-L1 expression: $26 \%$ response rate at with $\geq 5 \%$ expression, $18 \%$ with $\geq 1 \%$ to $<5 \%$ expression, and $15 \%$ overall response rate). Median survival time was 11.7 months, and median progression-free survival time was found to be 2.1 months regardless of PD-L1 expression status. In the $2^{\text {nd }}$ cohort of this study (NCT02108652), atezolizumab treatment resulted in an objective response rate of $16 \%$ in all patients and a $28 \%$ objective response rate in patients with $\geq 5 \%$ PD-L1 expression in TIMCs, after a median follow-up of 1.5 years $(21,22)$. The 12 -month overall survival rate of patients with $\geq 5 \%$ PD-L1 expression in TIMCs was 50\%, compared to $37 \%$ in the entire patient population $(21,22)$.

In terms of the adverse effect profile of atezolizumab in the IMvigor 211 trial (NCT02302807), 69\% of patients overall experienced a side effect. The most common adverse effects were fatigue (31\%), nausea (14\%), anorexia, pruritus, fever, diarrhea, rashes, and arthralgia. Pneumonia and dyspnea were serious side effects $(21,22)$. Based on these results, atezolizumab was approved by the FDA in May 2016 for the treatment of patients with locally advanced or metastatic urothelial carcinoma with disease progression during or after platinumbased chemotherapy. A study expected to be completed in the spring of 2018 (NCT02807636) is investigating the effect of atezolizumab \pm gemcitabine/carboplatin or chemotherapy with cisplatin alone on patients with locally advanced or metastatic urothelial carcinoma. Studies investigating the effects of atezolizumab in MIBC and NMIBC are presented in Tables 2 and 3 .

\section{Pembrolizumab (PD-1 Inhibitor)}

In February 2017, the FDA approved the evaluation of pembrolizumab as a first-line treatment in patients with urothelial carcinoma who are not eligible for cisplatin-based chemotherapy and as a second-line treatment in patients with urothelial carcinoma that progresses during or after platinumbased chemotherapy. In the phase $1 \mathrm{~b}$ trial investigating antiPD-1 pembrolizumab, 33 patients with recurrent or metastatic urothelial cancer were examined and 41\% PD-L1 expression was noted in the tumor cells (23). In the median follow-up period of 11 months, overall response and complete response rates of $24 \%$ and $10 \%$ were obtained. In the KEYNOTE-012 (NCT01848834) phase $1 \mathrm{~b}$ trial, the objective response rate was $25 \%$ and 12-month progression-free survival was $19 \%$ in the total population, while the objective response rate was $38 \%$ for tumors with positive PD-L1 expression (>1\% in tumor nests) (24). In KEYNOTE-012, side effects were observed in $61 \%$ of patients, and were most commonly reported as fatigue (18\%), peripheral edema (12\%), and nausea (9\%) (25). In KEYNOTE-052 (NCT02335424), a phase 2 study in which pembrolizumab was given as first-line therapy to patients with 
Celik et al.

\begin{tabular}{|c|c|c|c|c|c|c|c|c|c|c|c|c|c|c|c|c|c|c|}
\hline & 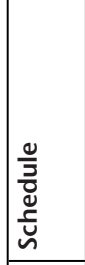 & 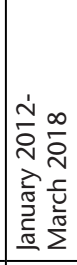 & 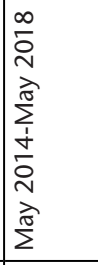 & 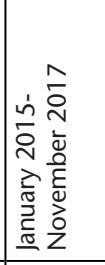 & 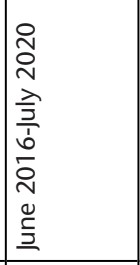 & 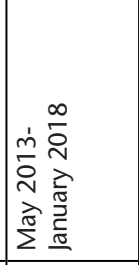 & 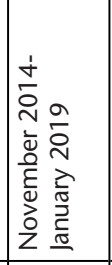 & 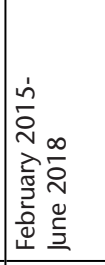 & 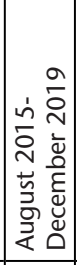 & 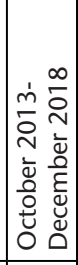 & 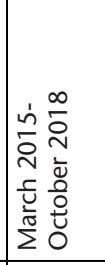 & 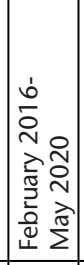 & 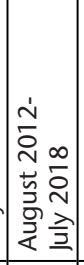 & 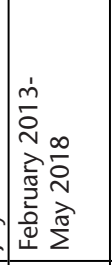 & 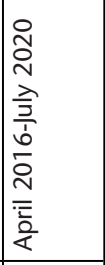 & 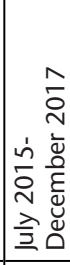 & 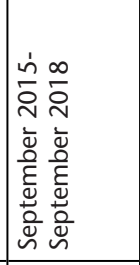 & 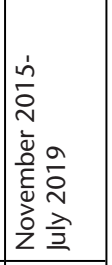 \\
\hline & 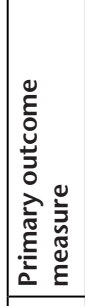 & 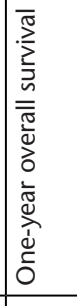 & 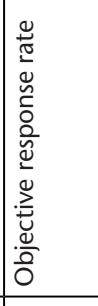 & 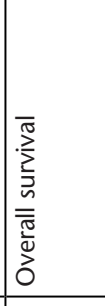 & 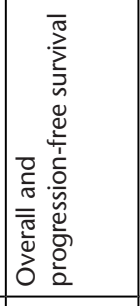 & 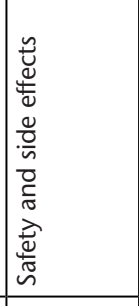 & 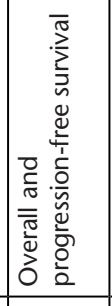 & 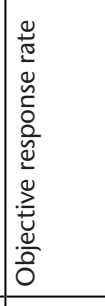 & 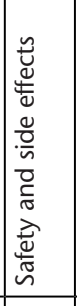 & 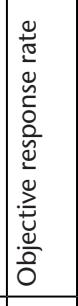 & 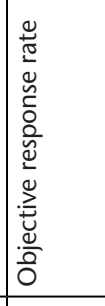 & 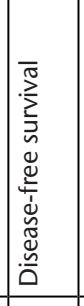 & 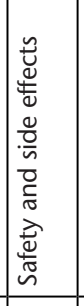 & 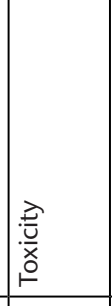 & 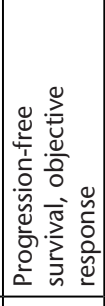 & 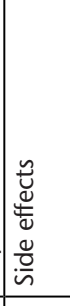 & 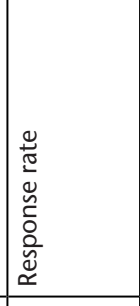 & 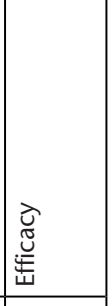 \\
\hline & $z$ & mి & $\frac{O}{m}$ & బ̃ & 오 & సे & 胥 & 总 & $\stackrel{\infty}{m}^{\infty}$ & 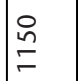 & 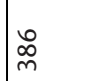 & 承 & 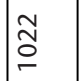 & 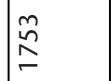 & 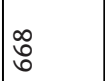 & 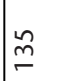 & I & 8 \\
\hline & $\begin{array}{l}\text { 芯 } \\
\frac{\tilde{c}}{\alpha} \\
\end{array}$ & N & N & $m$ & $\mathrm{~m}$ & - & $\mathrm{m}$ & $N$ & - & $\stackrel{2}{=}$ & N & $m$ & $\stackrel{\sqrt{2}}{=}$ & - & $m$ & - & $v$ & $m$ \\
\hline$\tilde{\xi}^{\widetilde{\sigma}}$ & $\begin{array}{l}\text {.0 } \\
\bar{y} \\
\text { صू }\end{array}$ & 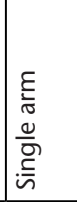 & 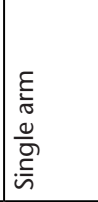 & 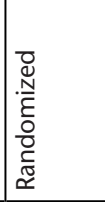 & 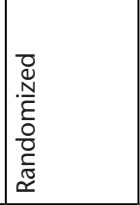 & 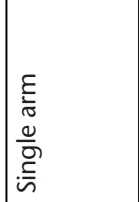 & 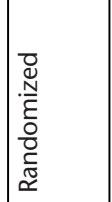 & 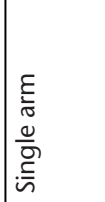 & 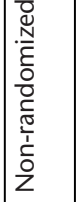 & 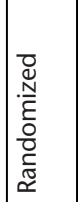 & 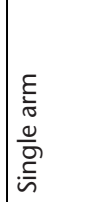 & 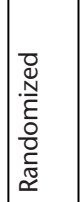 & 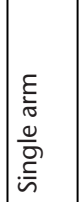 & 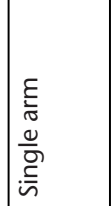 & 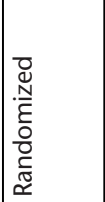 & 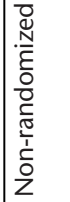 & 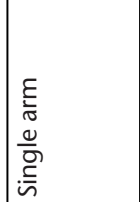 & 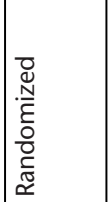 \\
\hline 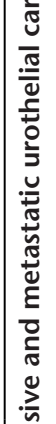 & $\begin{array}{l}t \\
0 \\
0 \\
0 \\
\end{array}$ & 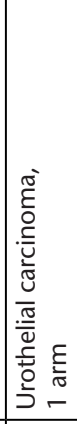 & 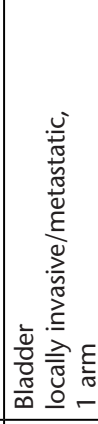 & 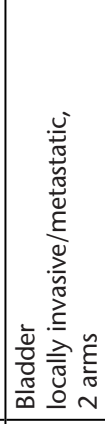 & 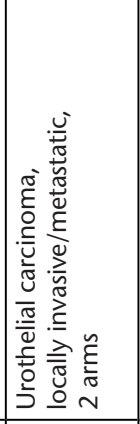 & 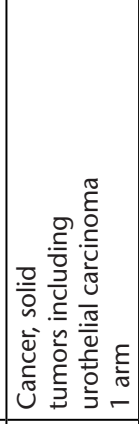 & 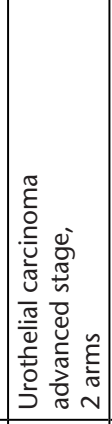 & 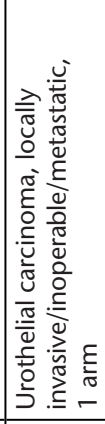 & 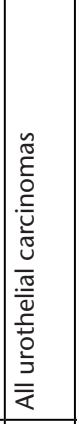 & 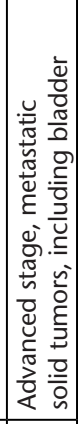 & 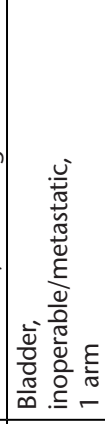 & 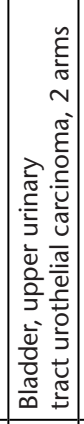 & 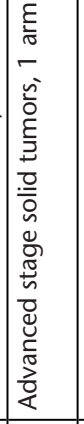 & 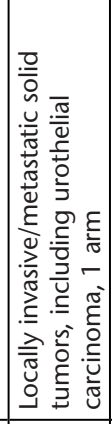 & 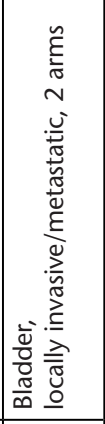 & 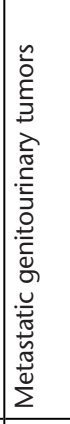 & 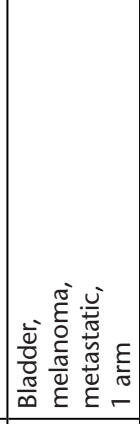 & 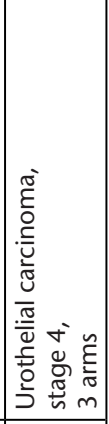 \\
\hline 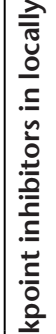 & 苞 & 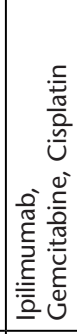 & 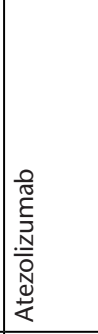 & 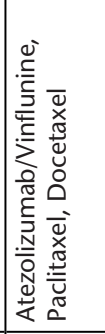 & 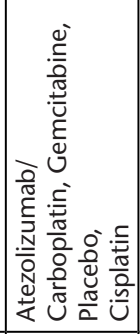 & 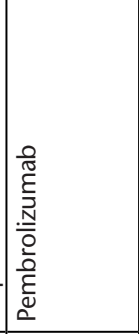 & 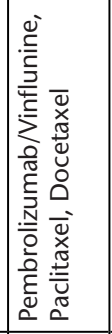 & 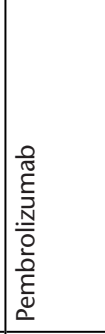 & 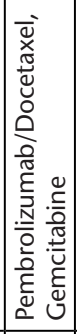 & 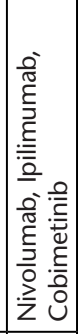 & 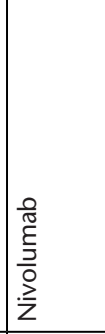 & 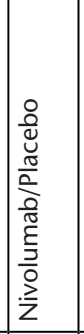 & 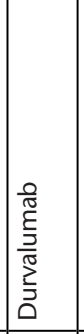 & 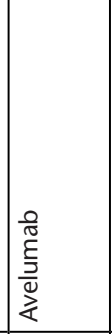 & 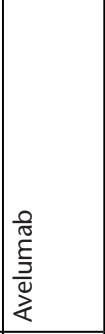 & 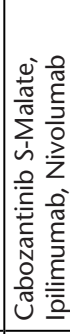 & 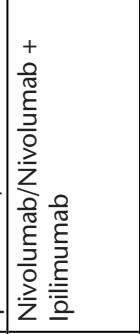 & 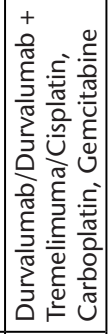 \\
\hline 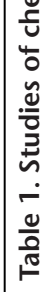 & 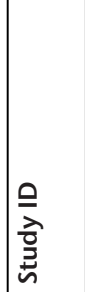 & 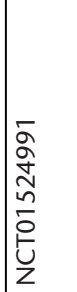 & 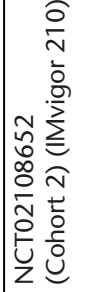 & 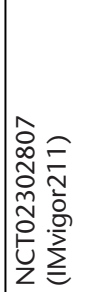 & 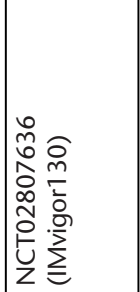 & 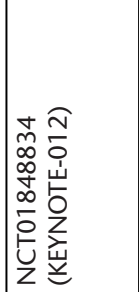 & 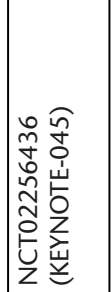 & 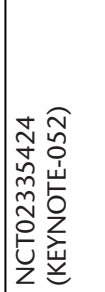 & 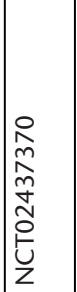 & 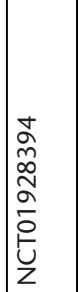 & 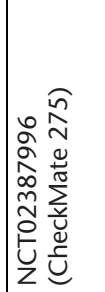 & 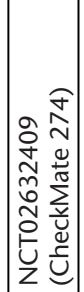 & $\begin{array}{l}0 \\
0 \\
0 \\
0 \\
0 \\
0 \\
0 \\
0 \\
z \\
z\end{array}$ & 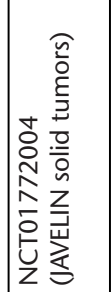 & 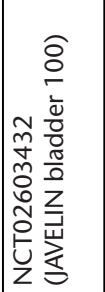 & 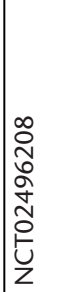 & 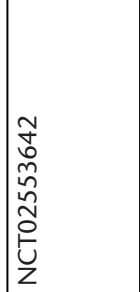 & 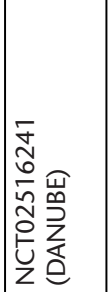 \\
\hline
\end{tabular}


advanced stage inoperable and metastatic urothelial carcinoma, the objective response rate was $24 \%$ in the first 100 patient analysis and $36.7 \%$ in patients whose PD-L1 expression rates in tumor and immune cells were $>10 \%(26)$. In the KEYNOTE-045 (NCT02256436) phase 3 trial, the overall survival in the chemotherapy and pembrolizumab randomization in patients with previously treated metastatic urothelial cancer was 10.3 months in the pembrolizumab arm and 7.4 months in the chemotherapy arm (27). Combinations of pembrolizumab with docetaxel or gemcitabine (NCT02437370) and gemcitabine or cisplatin (NCT02690558) are currently being investigated in ongoing studies. Combinations of pembrolizumab with chemotherapy and radiotherapy are also being investigated in studies currently in progress (NCT02662062 and NCT02621151). Studies investigating the effects of pembrolizumab in MIBC and NMIBC are presented in Tables 2 and 3.

\section{Nivolumab (PD-1 Inhibitor)}

Nivolumab is a monoclonal antibody against PD-1. Following its use in other types of cancer, nivolumab was approved by the FDA for use in the treatment of renal cell carcinoma in November 2015 and for use in patients with locally advanced or metastatic urothelial carcinoma progressing for one year after platinumbased chemotherapy in February 2017. In a trial including patients with metastatic urothelial cancer (NCT01928394), the objective response rates for patients with $\geq 1 \%$ and $<1 \%$ PD-L1 expression in tumor cells were $24 \%$ for $26 \%$, respectively, and overall survival for the entire population was 9.7 months (28). Approximately $21.8 \%$ of patients experienced grade 3-4 side effects, the most common of which were lipase elevation (5.1\%), amylase elevation (3.8\%), and fatigue (28). In the CheckMate 275 study (NCT02387996), an objective response rate of $19.6 \%$ was achieved with nivolumab in patients with metastatic urothelial cancer. The objective response rate was $16.1 \%$ for those with a low or negative PD-L1 expression in the tumor $(<1 \%)$, while this rate was $28.4 \%$ for those with $\geq 5 \%$ PD-L1 expression (29). CheckMate 274 (NCT02632409) is an ongoing phase 3 trial in which nivolumab is evaluated versus a placebo after surgery in patients with bladder or upper urinary tract cancer.

\section{Durvalumab (PD-L1 Inhibitor)}

Durvalumab, a monoclonal antibody against PD-L1, was evaluated by the FDA in February 2016 for patients with inoperable or metastatic urothelial bladder cancer that progressed during or after standard platinum-based chemotherapy.

In a phase 1/2 durvalumab trial involving patients with inoperable or metastatic urothelial bladder cancer (NCT01693562), the objective response rate was $31 \%$, while this rate was $0 \%$ in the low/negative PD-L1 subgroup $(<25 \%)$ and $46 \%$ in the high PD-L1 subgroup ( $\geq 25 \%)$ (30). The most common side effects observed in the study were fatigue (13\%), diarrhea (10\%), and decreased appetite (8\%).

\section{Avelumab (PD-L1 Inhibitor)}

This anti-PD-L1 monoclonal antibody is in the early stages of research for more than 15 types of cancer, including bladder cancer. Avelumab has a different mechanism than other PD-L1

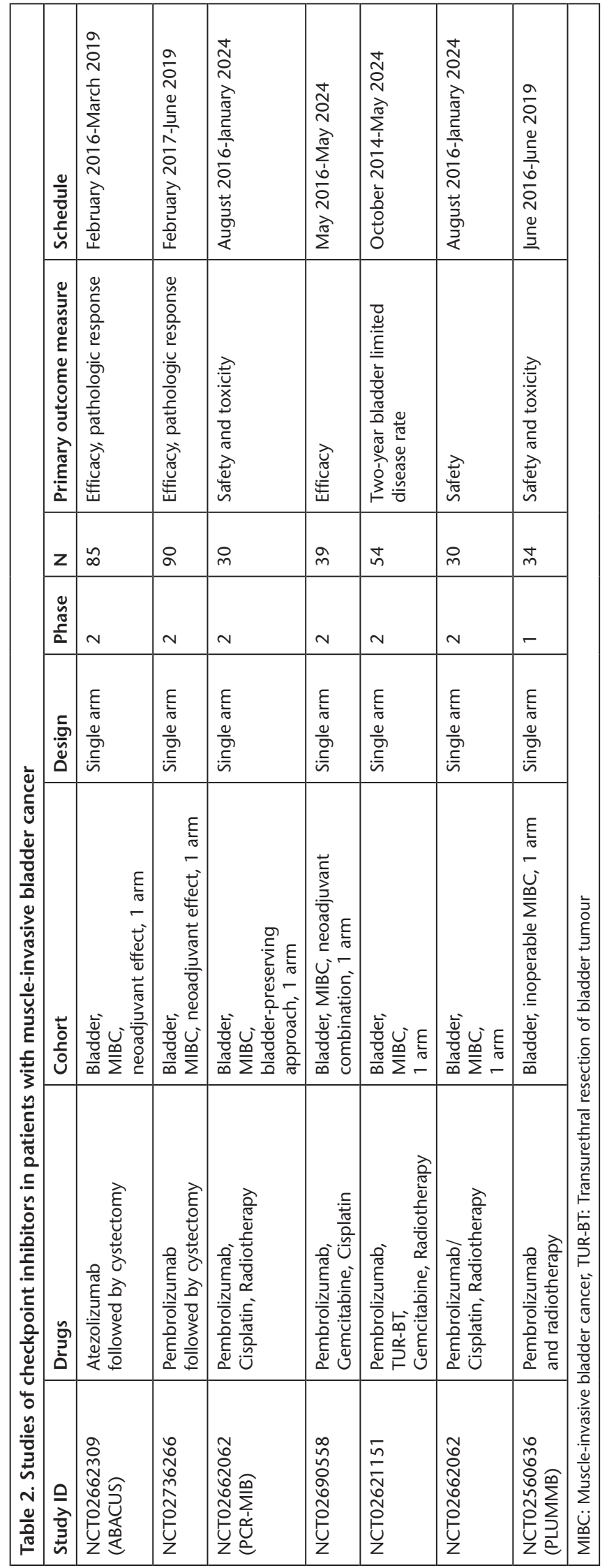


Çelik et al.

Checkpoint Inhibitors for Bladder Cancer

\begin{tabular}{|l|l|l|l|l|l|l|l|}
\hline \multicolumn{2}{|l|}{ Table 3. Studies of checkpoint inhibitors in patients with non-muscle-invasive bladder cancer } \\
\hline Study ID & Drugs & Cohort & Design & Phase & N & Primary outcome meausre & Schedule \\
\hline NCT02451423 & Atezolizumab & $\begin{array}{l}\text { Bladder, } \\
\text { NMIBC, } \\
\text { refractory to BCG, 1 arm }\end{array}$ & Single arm & 2 & 42 & Complete response rate & $\begin{array}{l}\text { April 2016- } \\
\text { December 2019 }\end{array}$ \\
\hline $\begin{array}{l}\text { NCT02625961 } \\
\text { (KEYNOTE-057) }\end{array}$ & Pembrolizumab & $\begin{array}{l}\text { Bladder, } \\
\text { high-risk NMIBC, } \\
\text { refractory to BCG, 1 arm }\end{array}$ & Single arm & 2 & 260 & $\begin{array}{l}\text { Complete response rate, } \\
\text { Disease-free survival }\end{array}$ & $\begin{array}{l}\text { February 2016- } \\
\text { December 2021 }\end{array}$ \\
\hline $\begin{array}{l}\text { NCT02324582 } \\
\text { (MARC) }\end{array}$ & $\begin{array}{l}\text { Pembrolizumab } \\
\text { and BCG }\end{array}$ & $\begin{array}{l}\text { Bladder, } \\
\text { high-risk NMIBC, } \\
\text { refractory to BCG, 1 arm }\end{array}$ & Single arm & 1 & 15 & Safety & $\begin{array}{l}\text { June 2015- } \\
\text { November 2020 }\end{array}$ \\
\hline BCG: Bacillus Calmette-Guérin, NMIBC: Non-muscle-invasive bladder cancer & & \\
\hline
\end{tabular}

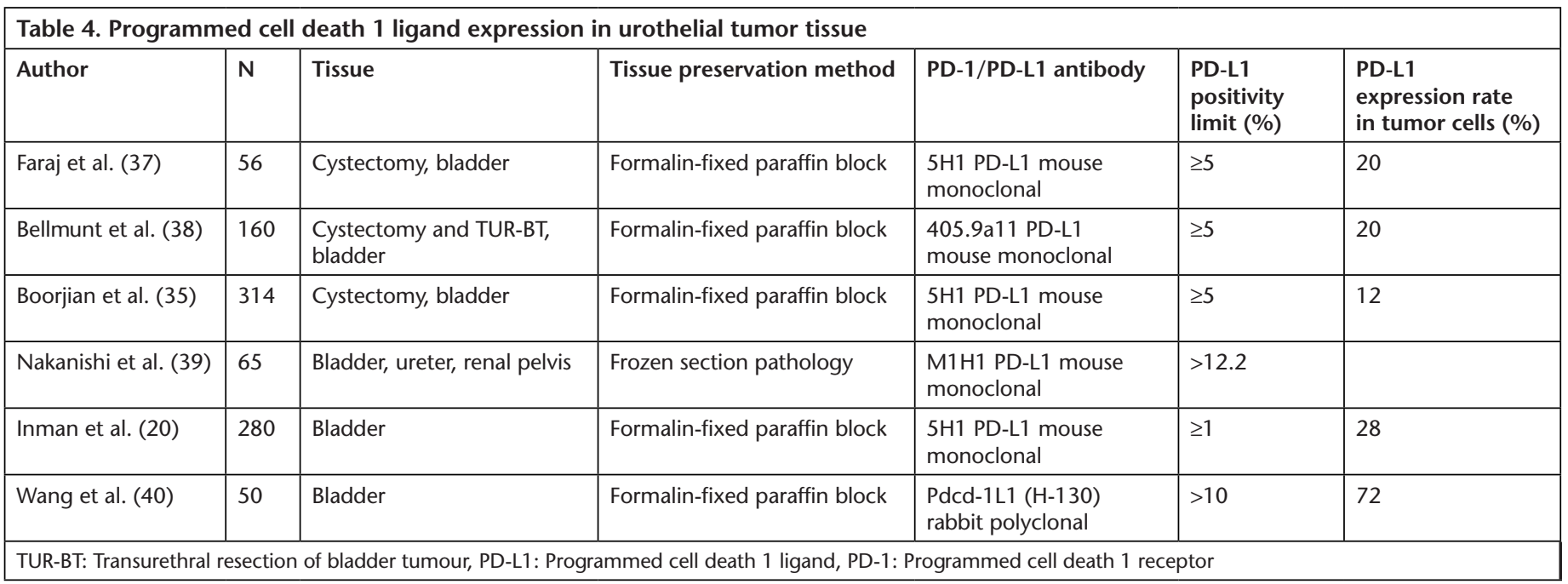

inhibitors. It exhibits antibody-dependent cellular cytotoxic activity in addition to PD-L1 inhibition. This causes the direct destruction of tumor cells, but this activity can also cause lysis of other cells expressing PD-L1 and lead to specific toxicities (31). In the JAVELIN solid tumor phase 1b study (NCT01772004), the objective response rate was $16.5 \%$ among patients whose cancer progressed after platinum-based chemotherapy or who were ineligible for platinum (32). Infusion-related reactions (22.5\%) and fatigue (14.7\%) were the most common side effects (32). The phase 3 JAVELIN bladder 100 study (NCT02603432) is currently ongoing.

\section{PD-1/PD-L1 and CTLA-4 Inhibitor Combination}

PD-1 is mainly involved in the effector phase of T-cell activation, and the PD-1/PD-L1 interaction primarily occurs during presentation of antigens to memory $T$ cells in peripheral tissues (33). CTLA-4 is expressed by regulatory $T$ cells and memory CD4 cells and functions during the early activation of $T$ cells found in lymphatic tissues (33). Therefore, the combination of treatments aimed at the inhibition of these two targets is logical.

The combination of nivolumab and ipilimumab is being investigated as part of the CheckMate 032 trial. Cohort $A(n=26)$ received a combination of nivolumab $(1 \mathrm{mg} / \mathrm{kg})$ and ipilimumab (3 $\mathrm{mg} / \mathrm{kg})$, cohort $B(n=104)$ received a combination of nivolumab (3 mg/kg) and ipilimumab (1 mg/kg) (34). A higher response rate was observed in the cohort with the higher dose ipilimumab (39\%) compared to the cohort with the lower dose (26\%) (34). However, overall survival time was similar in both groups (7.3 months versus 10.2 months) (34). Another ongoing study (NCT02553642) investigates PD-L1 expression and rates of response to nivolumab/ipilimumab combination therapy in patients with locally advanced/inoperable or metastatic urothelial carcinoma. Other studies evaluating combinations of nivolumab and ipilimumab (NCT01928394, NCT02496208) are summarized in Table 1.

The combination of durvalumab and tremelimumab, a CTLA-4 inhibitor, versus standard chemotherapy in patients with stage 4 urothelial bladder cancer is currently being assessed in the DANUBE trial (NCT02516241), expected to be completed in 2019.

In addition to these agents, $\mathrm{T}$ cell surface receptors such as B7-H3 and OX40, which regulate cell activation and efficacy, have also been discovered as potential therapeutic targets. Boorjian et al. (35) have suggested that high expression of the glycoprotein $\mathrm{B} 7-\mathrm{H} 3$ in urothelial tumors may be associated with upregulation of PD-1.

\section{The Role of Checkpoint Inhibitors in the Treatment of Muscle-Invasive Bladder Cancer}

Neoadjuvant MVAC (methotrexate, vincristine, adriamycin, cisplatin) or gemcitabine and cisplatin combinations are recommended regimens for MIBC. However, a large proportion of patients relapse after radical cystectomy (36). Furthermore, 
standard chemotherapy agents are not available for the patient group that is ineligible for platinum-based chemotherapy. Relevant studies involving checkpoint inhibitors and their combinations with chemotherapy and radiotherapy are in the research phase. Some of these studies are presented in Table 2. Besides these, the option of immunotherapy for MIBC patients who are ineligible for cystectomy or want bladder-preserving treatment is one of the current topics being discussed, and there are ongoing studies involving this patient group. Some of these studies are also shown in Table 2.

\section{The Role of Checkpoint Inhibitors in the Treatment of Non-Muscle-Invasive Bladder Cancer}

Research continues regarding the use of checkpoint inhibitors in the treatment of locally invasive and metastatic urothelial carcinoma, especially bladder cancer, and the role of these drugs in treatment. Their combinations with both neoadjuvant and adjuvant chemotherapy and chemo-radiotherapy, and the role of these combinations as first- and second-line therapies constitute a broad research area. A clearer picture is expected to develop in the 2020s. The next step for these treatments, which have already been investigated for NMIBC, involves studies targeting the $40 \%$ of the patient population that develops recurrence and progression into MIBC despite intravesical BCG therapy. Some relevant studies that are in progress, especially those involving BCG-refractory patient group, are listed in Table 3.

\section{PD-L1 Expression in Urothelial Tumor Tissue}

Studies examining PD-L1 expression levels in urothelial carcinoma have yielded differing results. These studies are briefly summarized in Table 4. In one of those studies, pathologic specimens of 56 patients who underwent radical cystectomy due to bladder cancer were examined and $\geq 5 \%$ PD-L1 expression was observed in $20 \%$ of them. However, it was shown that PD-L1 expression and cytotoxic CD8+ T cell density were not associated with the clinicopathologic data (37). Bellmunt et al. (38) reviewed the pathology specimens of 160 patients who underwent transurethral resection of bladder tumour (TUR-BT) or radical cystectomy and defined a threshold of $\geq 5 \%$ for PD-L1 positivity on tumor cells. Positive PD-L1 expression was detected in $40 \%$ of TIMCs and was associated with longer survival in metastatic disease (38). In a study of 314 cystectomy specimens, $\geq 5 \%$ PD-L1 expression was observed in urothelial tumor cells and the expression of PD-1 in TIMCs was markedly increased (35). In a study of 65 patients, $>12.2 \%$ PD-L1 expression was associated with high tumor grade and low recurrence-free survival (39). Another study demonstrated that increasing tumor stage was associated with higher PD-L1 expression positivity rate $(\geq 1 \%)(7 \%, 16 \%, 23 \%, 30 \%$, and $45 \%$ in $\mathrm{Ta}, \mathrm{T} 1, \mathrm{~T} 2, \mathrm{~T} 3 / 4$, and CIS tumors, respectively) (20). Yet another report stated that $>10 \%$ PD-L1 expression was associated with high-grade, muscle invasion, recurrence, and shorter survival (40). However, there are certain factors that make it difficult to direct compare these studies evaluating PD-L1 expression. These include differences in the organ sampled and collection method (TUR-BT, cystectomy, nephroureterectomy), differences in immunohistochemical analysis (formalin-fixed paraffin block vs. frozen tissue), different PD-L1 antibodies
(5H1, M1H1, and Pdcd-1L1) and differences in expression positivity rates (ranging from $1 \%$ to $12.2 \%)(20,35,39)$.

\section{Conclusion}

Although BCG is an important step in the treatment of NMIBC, additional treatments are needed in patients with treatment failure, as in locally invasive or metastatic bladder cancer. Of the immunotherapeutic agents investigated for this purpose, checkpoint inhibitors (CTLA-4, PD-1, and PD-L1 inhibitors) have provided favorable objective response rates and longer survival in locally advanced, inoperable, and metastatic bladder cancer, especially depending on expression levels in TIMCs and tumor cells. Although research priorities are inverse to disease severity, we look forward to the outcomes of ongoing studies in order to use these inhibitors in neoadjuvant, adjuvant, and bladder-preserving approaches to MIBC and in patients with BCG-refractory NMIBC.

\section{Questions}

1. What are the role and mechanism of action of Bacillus Calmette-Guerin in bladder cancer immunotherapy?

2. What is the role of checkpoints in the immune response and what are the effects of their inhibition?

3. What is the role of checkpoint inhibitors in the current treatment of bladder cancer?

4. What are the expectations regarding checkpoint inhibitors in the treatment of non-muscle invasive bladder cancer?

Ethics

Peer-review: Externally peer-reviewed.

\section{Authorship Contributions}

Concept: S.Ç., Design: S.Ç., Z.S.A., S.A., Data Collection or Processing: S.Ç., Analysis or Interpretation: S.Ç., Z.S.A., S.A., Literature Search: S.Ç., Writing: S.Ç.

Conflict of Interest: No conflict of interest was declared by the authors.

Financial Disclosure: The authors declared that this study received no financial support.

\section{References}

1. Howlader N, Noone AM, Krapcho M. SEER Cancer Statistics Review, April 2015 ed. Bethesda: National Cancer Institute; 1975-2012.

2. Babjuk $M$, Burger $M$, Compérat $E$, et al. European Association of Urology, Non-muscle-invasive Bladder Cancer Guideline, 2017.

3. Sylvester RJ, vander MA, Lamm DL. Intravesical bacillus CalmetteGuerin reduces the risk of progression in patients with superficial bladder cancer: a meta-analysis of the published results of randomized clinical trials. J Urol 2002;168:1964-1970.

4. Lawrence MS, Stojanov P, Polak, P et al. Mutational heterogeneity in cancer and the search for new cancer-associated genes. Nature 2013;499:214-218.

5. Donin NM, Lenis AT, Holden S, et al. Immunotherapy for the Treatment of Urothelial Carcinoma. J Urol 2017;197:14-22.

6. Morales A, Eidinger D, Bruce AW. Intracavitary bacillus CalmetteGuerin in the treatment of superficial bladder tumors. J Urol 1976;116:180-183.

7. Calmette $A$, Guerin $C$, Boquet $A$, et al. La Vaccination Preventive contre la Tuberculose par le "BCG". Paris: Masson et Cie 1927. 
8. Old LJ, Clarke DA, Benacerraf B. Effect of bacillus CalmetteGuerin infection on transplanted tumours in the mouse. Nature 1959;184:291-292.

9. deKernion JB, Golub SH, Gupta RK, et al. Successful transurethral intralesional BCG therapy of a bladder melanoma. Cancer 1975;36:1662-1667.

10. Lamm DL, Thor DE, Harris SC, et al. Bacillus Calmette-Guerin immunotherapy of superficial bladder cancer. J Urol 1980;124:38-40.

11. Redelman-Sidi G, Glickman MS, Bochner BH. The mechanism of action of BCG therapy for bladder cancerda current perspective. Nat Rev Urol 2014;11:153-162.

12. Powles T, Eder JP, Fine GD, et al. MPDL3280A (anti-PD-L1) treatment leads to clinical activity in metastatic bladder cancer. Nature 2014;515:558-562.

13. Petrylak DP, Powles T, Bellmunt J, et al. A phase la study of MPDL3280A (anti-PDL1): updated response and survival data in urothelial bladder cancer (UBC). J Clin Oncol 2015;33:4501.

14. Hodi FS, O'Day SJ, McDermott DF, et al. Improved survival with ipilimumab in patients with metastatic melanoma. $N$ Engl J Med 2010;363:711-723.

15. Kwon ED, Drake CG, Scher HI, et al. Ipilimumab versus placebo after radiotherapy in patients with metastatic castration-resistant prostate cancer that had progressed after docetaxel chemotherapy (CA184043): a multicentre, randomised, double-blind, phase 3 trial. Lancet Oncol 2014;15:700-712.

16. Carthon BC, Wolchok JD, Yuan J, et al. Preoperative CTLA-4 blockade: tolerability and immune monitoring in the setting of a presurgical clinical trial. Clin Cancer Res 2010;16:2861-2871.

17. Keir ME, Butte MJ, Freeman G], et al. PD-1 and its ligands in tolerance and immunity. Annu Rev Immunol 2008;26:677-704.

18. Pardoll DM. The blockade of immune checkpoints in cancer immunotherapy. Nat Rev Cancer 2012;12:252-264.

19. Dong H, Strome SE, Salomao DR, et al. Tumor associated B7H1 promotes T-cell apoptosis: a potential mechanism of immune evasion. Nat Med 2002;8:793-800.

20. Inman BA, Sebo TJ, Frigola $X$, et al. PD-L1 (B7-H1) expression by urothelial carcinoma of the bladder and BCG-induced granulomata: associations with localized stage progression. Cancer 2007; 109:1499-1505.

21. Rosenberg JE, Hoffman-Censits J, Powles T, et al. Atezolizumab in patients with locally advanced and metastatic urothelial carcinoma who have progressed following treatment with platinum-based chemotherapy: a single-arm, multicentre, phase 2 trial. Lancet 2016;387:1909-1920.

22. Loriot $\mathrm{Y}$, Rosenberg JE, Powles TB, et al. Atezolizumab (atezo) in platinum (plat)-treated locally advanced/metastatic urothelial carcinoma ( $\mathrm{mUC}$ ): updated OS, safety and biomarkers from the $\mathrm{Ph}$ II IMvigor210 study. European society for medical oncology. Copenhagen, Denmark: Proceedings; 2016. Abstr 783P.

23. Plimack ER, Gupta S, Bellmunt J, et al. A phase 1B study of pembrolizumab (Pembro; MK-3475) in patients with advanced urothelial tract cancer. Ann Oncol 2014;25:v1.

24. Plimack ER, Bellmunt J, Gupta S, et al. Pembrolizumab (MK-3475) for advanced urothelial cancer: Updated results and biomarker analysis from KEYNOTE-012. Chicago, IL: American Society of Clinical Oncology; 2015. Abstr 4502.

25. Gupta S, O'Donnell P, Plimack ER, et al. A phase 1b study of pembrolizumab (PEMBRO; MK-3475) for advanced urothelial cancer.. New Orleans, LA: American Urological Association; 2015. Abstr MP68-11.
26. Balar A, Bellmunt J, O’Donnell PH, et al. Pembrolizumab (pembro) as first-line therapy for advanced/unresectable or metastatic urothelial cancer: preliminary results from the phase 2 KEYNOTE-052 study. European society for medical oncology. Copenhagen, Denmark: Proceedings of ESMO; 2016. Abstr LBA32_PR.

27. Bellmunt J, De Wit R, Vaughn DJ, et al. Keynote-045: open-label, phase III study of pembrolizumab versus investigator's choice of paclitaxel, docetaxel, or vinflunine for previously treated advanced urothelial cancer. Society for Immunotherapy of Cancer (SITC) Annual Meeting. National Harbor, MD, 2016; Abstr 470.

28. Sharma $P$, Callahan MK, Bono $P$, et al. Nivolumab monotherapy in recurrent metastatic urothelial carcinoma (CheckMate 032): a multicentre, open-label, two-stage, multi-arm, phase 1/2 trial. Lancet Oncol 2016;17:1590-1598.

29. Galsky MD, Retz M, Siefker-Radtke AO, et al. Efficacy and safety of nivolumab monotherapy in patients with metastatic urothelial cancer (mUC) who have received prior treatment: Results from the phase II CheckMate 275 study. European Society for Medical Oncology. Copenhagen, Denmark 2016; Abstr LBA31_PR.

30. Massard C, Gordon MS, Sharma S, et al. Safety and efficacy of durvalumab (MEDI4736), an anti-programmed cell death ligand-1 immune checkpoint inhibitor, in patients with advanced urothelial bladder cancer. J Clin Oncol 2016;34:3119-3125.

31. Boyerinas B, Jochems C, Fantini M, et al. Antibody-dependent cellular cytotoxicity activity of a novel anti-PD-L1 antibody avelumab (MSB0010718C) on human tumor cells. Cancer Immunol Res 2015;3:1148-1157.

32. Patel MR, Ellerton J, Agrawal M, et al. Avelumab (MSB0010718C; antiPD-L1) in patients with metastatic urothelial carcinoma progressed after platinum-based therapy or platinum ineligible. European society for medical oncology. Copenhagen, Denmark: European Society for Medical Oncology; 2016. Abstr 777PD.

33. Ott PA, Hodi FS, Robert C. CTLA-4 and PD-1/PD-L1 blockade: new immunotherapeutic modalities with durable clinical benefit in melanoma patients. Clin Cancer Res 2013;19:5300-5309.

34. Sharma P, Callahan MK, Calvo E, et al. efficacy and safety of nivolumab plus ipilimumab in metastatic urothelial carcinoma: first results from the phase I/II checkmate 032 study. society for immunotherapy of cancer (SITC) annual meeting. National Harbor, MD, 2016; Abstr 449.

35. Boorjian SA, Sheinin Y, Crispen PL, et al. T-cell coregulatory molecule expression in urothelial cell carcinoma: clinicopathologic correlations and association with survival. Clin Cancer Res 2008;14:4800-4808.

36. Dash A, Pettus JA 4, Herr HW, et al. A role for neoadjuvant gemcitabine plus cisplatin in muscle- invasive urothelial carcinoma of the bladder: A retrospective experience. Cancer 2008; 113:2471-2477.

37. Faraj SF, Munari E, Guner G, et al. Assessment of tumoral PD-L1 expression and intratumoral CD8+ T cells in urothelial carcinoma. Urology 2015;85:7031-7036.

38. Bellmunt J, Mullane SA, Werner L, et al. Association of PD-L1 expression on tumor-infiltrating mononuclear cells and overall survival in patients with urothelial carcinoma. Ann Oncol 2015;26:812-817.

39. Nakanishi J, Wada Y, Matsumoto K, et al. Over expression of B7-H1 (PD-L1) significantly associates with tumor grade and postoperative prognosis in human urothelial cancers. Cancer Immunol Immunother 2007; 56:1173-1182.

40. Wang Y, Zhuang Q, Zhou S, et al. Costimulatory molecule B7-H1 on the immune escape of bladder cancer and its clinical significance. J Huazhong Univ Sci Technolog Med Sci 2009;29:77-79. 